\title{
Measuring Volitional Competences: Psychometric Properties of a Short Form of the Volitional Components Questionnaire (VCQ) in a Clinical Sample
}

\author{
Simon Forstmeier ${ }^{1, *}$ and Heinz Rüddel ${ }^{2}$ \\ ${ }^{I}$ Department of Psychology, Division of Psychopathology and Clinical Intervention, University of Zurich, Switzerland \\ and ${ }^{2}$ Psychosomatic Hospital St. Franziska-Stift, Bad Kreuznach, Germany
}

\begin{abstract}
Volitional competences (skills of will), including self-regulation skills such as self-motivation and emotion regulation and self-control skills such as impulse control, are particularly necessary for patients with psychiatric and psychosomatic disorders. The Volitional Components Questionnaire (VCQ) is an instrument designed to measure volitional competences. However, its length of 190 items prevents its routine application in clinical settings. This study evaluates a new 36-item short form of the VCQ. 1018 inpatients of a psychosomatic rehabilitation clinic completed the VCQ and several measures of psychopathology, personality, and cognitive ability. Exploratory factor analysis identified six factors. Confirmatory factor analysis showed that the VCQ-36 shared several volitional components with the original VCQ. Most of the self-regulation competences correlated negatively with psychopathological measures such as depression, as well as with neuroticism, social inhibitedness, and excitability, and positively with extraversion. Impulse control was also negatively associated with neuroticism and excitability. No meaningful correlation with cognitive ability was observed. The VCQ-36 is a reliable and valid instrument for assessing volitional competences and is well suited for routine application in clinical settings.
\end{abstract}

\section{INTRODUCTION}

The basic constituent in most approaches to volition is the human capacity to regulate other psychological functions such as emotion, motivation, and cognition in order to reach challenging goals that require some sort of volitional regulation. Several theories of volition have been proposed, with labels such as willpower [1], ego strength [2], action orientation [3, 4], self-regulation [5-10], self-control [7, 8, 11], and volition [12-14].

Some theoretical conceptions focus on single volitional competences without directly referring to volition as the superordinate concept (e.g., motivation regulation [15, 16], emotion regulation [17-21], and attention regulation [22, 23]). In his theory of self-regulation, Kuhl [7, 8] offers a comprehensive approach to the variety of volitional competences that are utilized by an individual to regulate other psychological functions. This theory forms the volitional part of a broader theory of volitional action regulation [9, 14]. Volition, or the will, is conceptualised as the central control instance which coordinates mental processes and subsystems in a way that the implementation of intentions is optimized. The most important mental subsystems that have to be coordinated according to an intention are attention, motivation, emotion, activation, cognition, and behaviour. Volition is

\footnotetext{
*Address correspondence to this author at the Department of Psychology, Division of Psychopathology and Clinical Intervention, University of $\mathrm{Zu}-$ rich, Binzmuehlestr. 14/17, 8050 Zurich, Switzerland; Tel: ++41 (0)44 635 73 05; Fax: ++41 (0)44 63573 19;

E-mail: s.forstmeier@psychologie.uzh.ch
}

differentiated into several volitional competences, which can be described as either consciously deployable strategies or unconsciously represented mechanisms.

The volitional competences are grouped into two basic modes of volition: self-regulation and self-control. Kuhl [7, $8,27]$ conceptualizes self-control as the self-disciplining mode of volition. Subsystems such as motivation or emotion that are at variance with the active intention are inhibited. Self-control comprises goal recollection, forgetfulness prevention, planning skill, impulse control, and initiating control. Self-regulation is conceptualized as the self-integrating mode of volition. Subsystems interact and are modified in order to unite as many subsystems as possible behind an intention. Self-regulation comprises attentional focusing, selfmotivation, emotion regulation, self-activation, self-relaxation, decision regulation, and coping with failure.

Although theories of volition have mainly been developed in the field of basic psychology, they have been extensively applied in clinical, health, educational, and organizational psychology. Volitional competence promotes recovery from stress [24] and correlates with positive attitudes (e.g., self-confidence, optimism) and with fewer reports of anxiety and depression $[6,11,25]$. Deficits in volitional competence are associated with various psychiatric disorders [26-28] and are probably a vulnerability factor for anxiety disorders [29] and depression [30]. Self-regulation is also associated with better social relationships and interpersonal skills [11, 31]. Volitional competences predict studying behaviour [32], higher school grades [11], and enactment of transfer intentions in management training [33]. 


\section{Measures of Volition}

Several measures of volition have been proposed, including the Action Control Scale (ACS) [34], the Locomotion and Assessment Questionnaire (LAQ) [6], the Self-Control Scale (SCS) [11], the Self-Regulation Scale (SRS) [25], and the Emotion Regulation Questionnaire (ERQ) [17]. However, only the Volitional Components Questionnaire (VCQ) by Kuhl [27] measures the variety of volitional competences conceptualized in the theory of self-regulation [7,8]. The VCQ is available as a long (VCQ-L) and short form (VCQS) [35]. The VCQ-L consists of 38 scales (5 items per scale) making up four sections. Fourteen scales measure the volitional competences that constitute the two modes of volition (nine self-regulation and five self-control scales). Thirteen scales assess symptoms of reduced access to these volitional competences under conditions of frustration or stress ("state orientation"). Two scales measure volitional and general self-confidence. Finally, nine scales assess symptoms of spontaneous control without volitional activity ("volition avoidance"). The test person rates the extent to which each item applies to him-/herself on a 4-point Likert scale $(0=$ not applicable, $3=$ wholly applicable). The internal consistency of the scales is reported to be moderate to high (Cronbach's $\alpha$ between .67 and .90) [36]. Norm values are available separately for women and men (obtainable from the test authors).

The VCQ-S, the official short form, consists of 12 scales selected from the 38 scales of the VCQ-L [35]. Only some of the volitional competences identified can be measured with this instrument: five of the nine self-regulation competences (self-determination, attentional focusing, self-motivation, self-relaxation, coping with failure) and two of the five selfcontrol competences (planning skill and initiating control).

The external validity of the VCQ-L and VCQ-S is supported by a variety of studies [36]. First, several studies have shown that the instruments predict the enactment of difficult behaviours requiring self-regulation or self-control, such as children's resistance to temptation (see [27]), the implementation of therapy intentions [28, 37], the enactment of transfer intentions in management training [33], and studying behaviour [32]. Second, inhibited access to one's own feelings ("alienation") correlates positively with VCQ scales measuring introjection and external control of action, and negatively with scales measuring self-determination $[35,36]$. Third, the validity of the VCQ is supported by studies showing the negative health outcomes of deficits in self-regulation $[27,28]$.

\section{The Present Study}

The aim of the present study is to construct a short form of the VCQ that fulfils two criteria. First, it should require not more than 10 minutes to complete, making it suitable for use in routine clinical care. Second, it should cover almost all volitional competences postulated in the theory of selfregulation [7, 8] and assessed with the VCQ-L [27]. The problem with the existing VCQ-S is that it omits three important self-regulation competences (self-activation, emotion regulation, decision regulation) and three equally important self-control competences (goal recollection, forgetfulness prevention, impulse control). These scales are essential to a measure of volitional competence for theoretical and clinical reasons: emotion and decision regulation as well as impulse control are among the major domains of volitional control [11] and are relevant in clinical practice. Emotion regulation is one of the main targets of psychotherapy, self-activation and decision regulation are important outcome variables in the treatment of depression, and impulse control is central in disorders involving over-control of impulses (e.g., obsessivecompulsive disorder, anorexia) or under-control of impulses (e.g., substance abuse, binge-eating disorder). In conclusion, there is a need for a new short form of the VCQ covering the whole range of volitional competences.

Therefore, the present study was aimed at developing a short form of the VCQ that (a) is confined to the scales measuring volitional competences, and (b) reduces the number of items per scale. This paper reports the psychometric properties of a 36-item version of the VCQ, its factorial structure as assessed by exploratory and confirmatory factor analysis, and the relation of the volitional components to other constructs including psychopathology, personality, and cognitive ability. We chose to test the instrument's psychometric properties in a clinical sample in order to replicate previous findings (e.g., [27]) in samples of psychosomatic inpatients.

Previous findings have shown that self-regulation competences are related to more positive emotions, fewer negative emotions, and less psychopathology, while self-control competences exhibit the opposite pattern [38]. Therefore, we expected to find meaningful negative correlations between psychopathology and self-regulation competences, and positive correlations between psychopathology and self-control competences. No such association was expected for physical complaints. Because of their associations with affectivity, self-regulation competences were also expected to show negative associations with personality characteristics such as neuroticism, social inhibitedness, and excitability, and a positive association with extraversion. These hypotheses can also be derived from the results of the VCQ-L [27]. Although we did not expect to find a meaningful correlation with cognitive ability (see [27]), we expected self-regulation (but not self-control) to be associated with achievement orientation. Finally, as found with the VCQ-L, we expected higher volitional competences in men than in women [27].

\section{METHOD}

\section{Sample and Procedures}

All inpatients $(n=1590)$ admitted to a psychosomatic rehabilitation centre between April 2002 and September 2003 were invited to participate after being given information about the study. 1018 patients agreed to participate (64\%). The psychiatric and psychosomatic disorders treated in the clinic were affective, anxiety, eating, adjustment, and somatoform disorders as well as tinnitus and obesity, diagnosed according to ICD-10 (World Health Organization, [39]). Not admitted to the clinic were patients with drug or alcohol addiction, acute risk of suicide, brain disorder or acute psychosis. The patients completed all questionnaires in two sessions on the second and third day after their admission. All patients provided written informed consent, and the study protocol was approved by the ethics committees of the regional medical authority. 


\section{6-Item Short Form of the VCQ (VCQ-36)}

As described in the introduction, the VCQ-L is made up of four sections, one of which assesses fourteen volitional competences. The self-regulation competences measured are attentional focusing (item example: "If I want to, I am able to deliberately concentrate on whatever is important at the moment"), self-motivation (e.g., "I can usually motivate myself quite well if my determination to persevere weakens"), emotion regulation (e.g., "I can deliberately think of pleasant things in order to make much better headway"), selfactivation (e.g., "I often get really activated when something is difficult"), self-relaxation (e.g., "I am able to relax quickly even after some inner tension"), decision regulation (e.g., "When I think about doing or not doing something, I usually arrive at a decision quickly"), and coping with failure (e.g., "I usually have to repeat a mistake in order to avoid making it once and for all"). The self-control competences measured are goal recollection (e.g., "As long as I have not settled a matter, I repeatedly remind myself of the things I want do"), forgetfulness prevention (e.g., "I often use memory aids in order to do what I intend to do at the appropriate time"), planning skill (e.g., "Before starting on something new, I usually make a plan"), impulse control (e.g., "When a temptation arises, I often feel defenceless"), and initiating control (e.g., "If a task has to be finished, I prefer to start it immediately"). These twelve scales, each of five items, result in a total of 60 items. Two further scales, unconscious attention control and self-determination, were omitted because their content was thought to be covered by other scales (e.g., attentional focusing and self-motivation). Validity and reliability of all scales are described in the introduction. In order to develop the 36-item short form, we selected the three items with the highest item-scale correlation from each of the twelve scales. Item and scale characteristics of the VCQ-36 are reported in the results section.

\section{External Criteria}

Depression was assessed by the Center for Epidemiological Studies Depression Scale (CES-D) [40] in its German version [41], a widely used self-report scale measuring depressive symptoms. Total psychiatric symptomatology was measured by the Symptom Checklist-9 (SCL-9) [42], a German short form of the widely used SCL-90-R [43]. For the construction of the unidimensional SCL-9, one item of each SCL-90-R scale with the highest correlation with the Global Severity Index (GSI), the mean of all nine original SCL-90$\mathrm{R}$ scales, was chosen. Physical complaints were assessed by the Giessen Subjective Complaints List (Giessener Beschwerdebogen, GBB) [44], with the five scales fatigue, stomach trouble, pain, dyscardia, and a total value for physical complaints. Personality structure was assessed using the Freiburg Personality Inventory (Freiburger PersönlichkeitsInventar, FPI) [45], a personality questionnaire frequently used in Germany. Five scales were selected on the basis of our hypotheses: achievement orientation, inhibitedness, excitability, extraversion, and neuroticism. The Digit Connection Test (Zahlen-Verbindungs-Test, ZVT) [46] was used to assess the basal cognitive speed underlying all intelligent performances. This is largely equivalent to the speed component of common intelligence tests.

\section{Data Analysis}

Descriptive statistics include means, standard deviations, Cronbach's $\alpha$ (internal consistency), adjusted item-score correlations, skewness and kurtosis of scales. Sub-sample and sex differences were tested using $t$-tests or $\chi^{2}$-tests. For the application of exploratory factor analysis (EFA) and confirmatory factor analysis (CFA), the sample was divided into two halves. EFA was used to examine the factor structure of the new VCQ-36, with parallel analysis (PA) [47] as extraction method. As an empirically supported and objective method for determining the most optimal number of factors to extract, PA is widely recommended by statisticians [48].

Four CFA models were tested for the variance-covariance matrix using the AMOS 6.0 statistical package. The maximum likelihood method was used, and correlations between the latent variables (factors) were included. The main estimates of a model's fit to the data were the root mean square error of approximation (RMSEA) and the standardized root mean square residual (SRMR). RMSEA values of .06 or less are said to reflect a model with a good fit to the data, and values between .06 and .08 to reflect an adequate fit [49]. SRMR values of .06 and less suggest a good fit to the data, while values between .06 and .11 suggest an adequate fit [49]. We also report comparative fit indices, the Akaike information criterion (AIC) and the Bayes information criterion (BIC). If the standard fit indices (RMSEA and SRMR) are acceptable, the model with the lowest AIC and $\mathrm{BIC}$ is to be preferred.

To investigate the association between volitional competences and external criteria, we calculated zero-order correlations. Because many significant correlations were to be expected given the large sample, only correlations with medium or high effect sizes $(r>=.25)$ were interpreted as meaningful.

\section{RESULTS}

\section{Characteristics of the Sample}

Table 1 shows the characteristics of the sample.

\section{Descriptive Statistics}

Table 2 shows means and standard deviations of the items as well as adjusted item-scale correlations for the original VCQ-L and the new VCQ-36. The item-scale correlations ranged between .40 and .75 . Table 3 presents means, standard deviations, and internal consistencies of the VCQ36 scales. Females showed significantly lower scores on most scales. Cronbach's $\alpha$ coefficients ranged between .56 and .92 , with most lying above .75 . Skewness and kurtosis values were in the -1 to +1 interval, indicating that all scales had a normal and symmetrical distribution.

\section{Differences between Disorders}

As Table 1 shows, depressive disorders are the most frequent disorders in this sample. In fact, $55 \%$ of the sample has a depressive syndrome (incl. depressive episode, recurrent depressive disorder, and dysthymia; see Table 4). The question arises as to whether depressed and non-depressed patients differ on VCQ-36 scales. Table 4 presents the means of the VCQ-36 scales for all disorder-related subsamples. 
Table 1. Demographic Characteristics of the Sample $(\mathrm{N}=1018)$

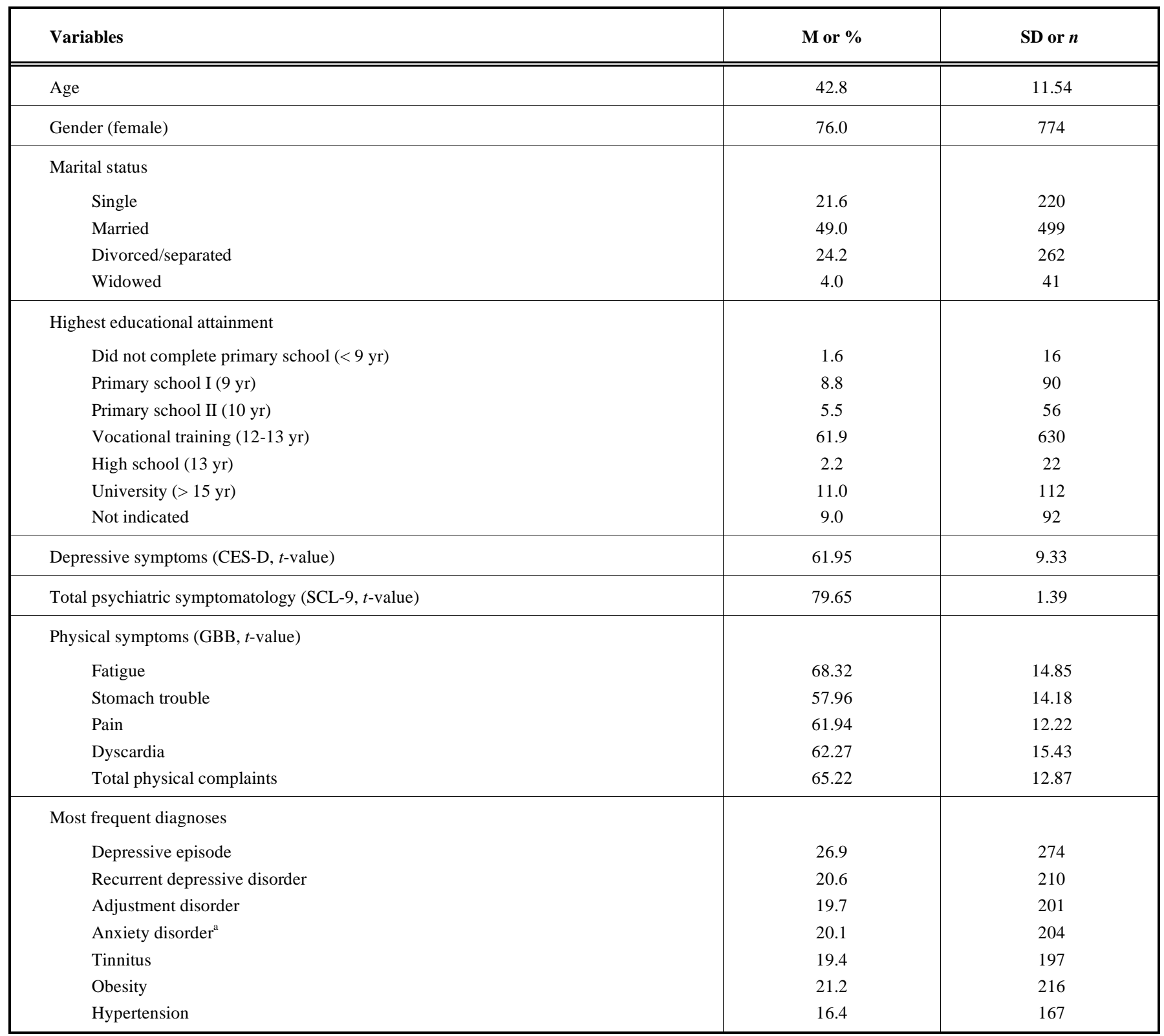

${ }^{a}$ Anxiety disorders involves agoraphobia, social phobia, specific phobia, panic disorder, and generalized anxiety disorder.

There were no significant differences between most nondepressed subsamples and depressed patients. Patients with phobic disorders exhibit significantly lower self-regulation skills, but not self-control skills, than depressed patients. By contrast, patients with adjustment disorders have significantly higher self-regulation and self-control skills, i.e., they are less impaired in volition than depressed patients. Finally, impulse control is especially highly impaired in patients with bulimia nervosa and overeating, but not in patients with anorexia nervosa.

\section{Exploratory Factor Analyses}

In order to examine construct validity, a PCA of the 36 items with Varimax rotation was carried out using one half of the sample, after previously measuring sample adequacy by means of the Kaiser-Meyer-Olkin test $(\mathrm{KMO}=.917)$ and Bartlett's test of sphericity (approx. $\chi^{2}(630)=8429.995 ; p<$ $.001)$. Using parallel analysis as extraction method, we identified six significant factors, which, taken together, accounted for $56.36 \%$ of the total variance (see Table 5). Factor 1 , labelled motivation, emotion, and relaxation regulation, contains all items of the self-motivation, emotion regulation, and self-relaxation scales, plus one impulse control item (Im3) and one attentional focusing item (At1), both of which also concern the regulation of inner processes. Factor 2, labelled planning and goal recollection, consists of all items of these two scales. Factor 3, labelled decision, activation, and attention regulation, consists of all items of the decision regulation and self-activation scales, plus two attentional focusing items that relate to starting action with one's full concentration. Factor 4, labelled coping and impulse 
Table 2. Item Characteristics: Means, Standard Deviations, and Item-Scale Correlations $(\mathbf{N}=1018)$

\begin{tabular}{|c|c|c|c|c|c|}
\hline & Attentional focusing & & & & \\
\hline At2 & Deliberately concentrating on task-relevant information & 1.56 & .83 & .65 & .70 \\
\hline Mo1 & Knowing how to increase pleasure in something & .94 & .78 & .64 & .61 \\
\hline Mo2 & Motivating oneself to persevere & 1.15 & .78 & .63 & .59 \\
\hline Mo3 & Finding a way to make an activity fun again & .96 & .74 & .60 & .57 \\
\hline \multirow[t]{2}{*}{ Em3 } & Overcoming an unpleasant mood & .89 & .73 & .65 & .62 \\
\hline & Self-activation & & & & \\
\hline Ac1 & Getting activated on difficult tasks & 1.19 & .91 & .76 & .75 \\
\hline Ac2 & Getting into best form when difficulties arise & 1.04 & .97 & .74 & .75 \\
\hline \multirow[t]{2}{*}{ Ac3 } & Becoming more active when obstacles arise & 1.18 & .88 & .65 & .63 \\
\hline & Self-relaxation & & & & \\
\hline $\operatorname{Re} 1$ & Relaxing quickly after inner tension & .63 & .72 & .67 & .67 \\
\hline \multirow[t]{2}{*}{ De3 } & Feeling clearly that a decision was correct & 1.44 & .83 & .55 & .53 \\
\hline & Coping with failure & & & & \\
\hline Co1 & Repeating a mistake (-) & 1.18 & .89 & .64 & .63 \\
\hline $\mathrm{Co} 2$ & Learning from mistakes quickly & 1.49 & .84 & .63 & .57 \\
\hline \multirow[t]{2}{*}{$\mathrm{Co} 3$} & Not being able to change behaviour quickly (-) & 1.27 & .83 & .63 & .62 \\
\hline & Goal recollection & & & & \\
\hline Go1 & Repeatedly reminding myself of things to do & 1.59 & .85 & .54 & .56 \\
\hline Go2 & Rehearsing the things to do & 1.79 & .77 & .53 & .54 \\
\hline \multirow[t]{2}{*}{ Go3 } & Keeping non-finished things in mind & 1.87 & .74 & .49 & .54 \\
\hline & Forgetfulness prevention & & & & \\
\hline Fo1 & Using memory aids & 1.69 & .96 & .42 & .51 \\
\hline Fo2 & Needing no memory aids (-) & 1.11 & .90 & .40 & .40 \\
\hline Fo3 & Writing down important things & 2.02 & .85 & .42 & .52 \\
\hline
\end{tabular}


(Table 2) contd....

\begin{tabular}{|c|c|c|c|c|c|}
\hline & Planning skill & & & & \\
\hline $\mathrm{P} 12$ & Determining the procedure before starting & 1.74 & .84 & .69 & .69 \\
\hline $\operatorname{Im} 1$ & Feeling defenceless when exposed to temptation (-) & 1.11 & .81 & .50 & .48 \\
\hline $\operatorname{Im} 2$ & Inability to resist a sudden impulse (-) & 1.28 & .78 & .48 & .48 \\
\hline $\operatorname{Im} 3$ & Ability to suppress everything in me & 1.13 & .81 & .39 & .36 \\
\hline In3 & Beginning something without hesitation & 1.61 & .88 & .67 & .65 \\
\hline
\end{tabular}

Notes: *The full set of VCQ items in German and English can be obtained from Prof. Julius Kuhl, University of Osnabrück, Germany. Range 0-3 (0 = not applicable, $3=$ wholly applicable); (-) inverted item; $r$ it-sc (VCQ-L): adjusted item-scale correlations in the original VCQ; $r$ it-sc (VCQ-36): adjusted item-scale correlations in the new VCQ-36.

Table 3. Scale Characteristics of the VCQ-36: Means, Standard Deviations, and Internal Consistency

\begin{tabular}{|c|c|c|c|c|c|c|c|c|c|c|}
\hline \multirow{2}{*}{ Scale } & \multicolumn{3}{|c|}{ Total $(N=1018)$} & \multicolumn{3}{|c|}{ Female $(N=774)$} & \multicolumn{3}{|c|}{ Male $(N=244)$} & \multirow{2}{*}{$\begin{array}{c}\text { Sex diff. } \\
t \text {-test }\end{array}$} \\
\hline & $\mathbf{M}$ & SD & $\alpha$ & $\mathbf{M}$ & SD & $\alpha$ & $\mathbf{M}$ & SD & $\alpha$ & \\
\hline Self-regulation & 3.57 & 1.52 & .92 & 3.43 & 1.46 & .91 & 4.00 & 1.62 & .93 & $-5.10 * * *$ \\
\hline Attentional focusing & 4.37 & 2.14 & .82 & 4.28 & 2.14 & .82 & 4.63 & 2.13 & .82 & $-2.18 *$ \\
\hline Self-motivation & 3.05 & 1.89 & .76 & 2.92 & 1.86 & .74 & 3.47 & 1.90 & .79 & $-4.01 * * *$ \\
\hline Emotion regulation & 2.66 & 1.88 & .79 & 2.53 & 1.85 & .79 & 3.09 & 1.92 & .80 & $-4.05 * * *$ \\
\hline Self-activation & 3.41 & 2.42 & .84 & 3.26 & 2.41 & .85 & 3.88 & 2.38 & .83 & $-3.50 * * *$ \\
\hline Self-relaxation & 2.05 & 1.85 & .80 & 1.87 & 1.74 & .78 & 2.63 & 2.05 & .84 & $-5.21 * * *$ \\
\hline Decision regulation & 4.40 & 2.11 & .74 & 4.31 & 2.09 & .72 & 4.69 & 2.14 & .79 & $-2.46^{* *} *$ \\
\hline Coping with failure & 5.04 & 2.13 & .77 & 4.86 & 2.11 & .77 & 5.60 & 2.09 & .77 & $-4.77 * * *$ \\
\hline Self-control & 5.09 & 1.22 & .82 & 5.06 & 1.25 & .82 & 5.18 & 1.14 & .80 & -1.29 \\
\hline Goal recollection & 5.25 & 1.90 & .73 & 5.29 & 1.92 & .73 & 5.11 & 1.85 & .70 & 1.32 \\
\hline Forgetfulness prevention & 5.59 & 2.02 & .60 & 5.69 & 2.04 & .61 & 5.27 & 1.92 & .57 & $2.83 * *$ \\
\hline Planning skill & 4.94 & 2.22 & .81 & 4.77 & 2.27 & .81 & 5.50 & 1.95 & .77 & $-4.93 * * *$ \\
\hline Impulse control & 4.74 & 1.78 & .59 & 4.62 & 1.79 & .59 & 5.13 & 1.70 & .58 & $-3.93 * * *$ \\
\hline Initiating control & 4.92 & 2.38 & .84 & 4.94 & 2.39 & .84 & 4.87 & 2.36 & .85 & .36 \\
\hline
\end{tabular}

Notes: Range 0-9; $\alpha$ : Cronbach's alpha; testing gender difference using t-tests for independent samples.

$* p<.05 . * * p<.01$. *** $p<.001$.

control, contains all items of the coping with failure scale and two impulse control items. Factors 5 and 6, labelled initiating control and forgetfulness prevention, respectively, contain items from the respective original scales (see Table 5).

\section{Confirmatory Factor Analyses}

Four models for the VCQ-36 were tested: (1) a 12-factor model with the 12 original VCQ-36 scales (Table 2 ); (2) a 6- 
Table 4. VCQ-36 Scale Means for Subsamples with ICD-10 Mental and Behavioural Disorders $(\mathrm{N}=1018)$

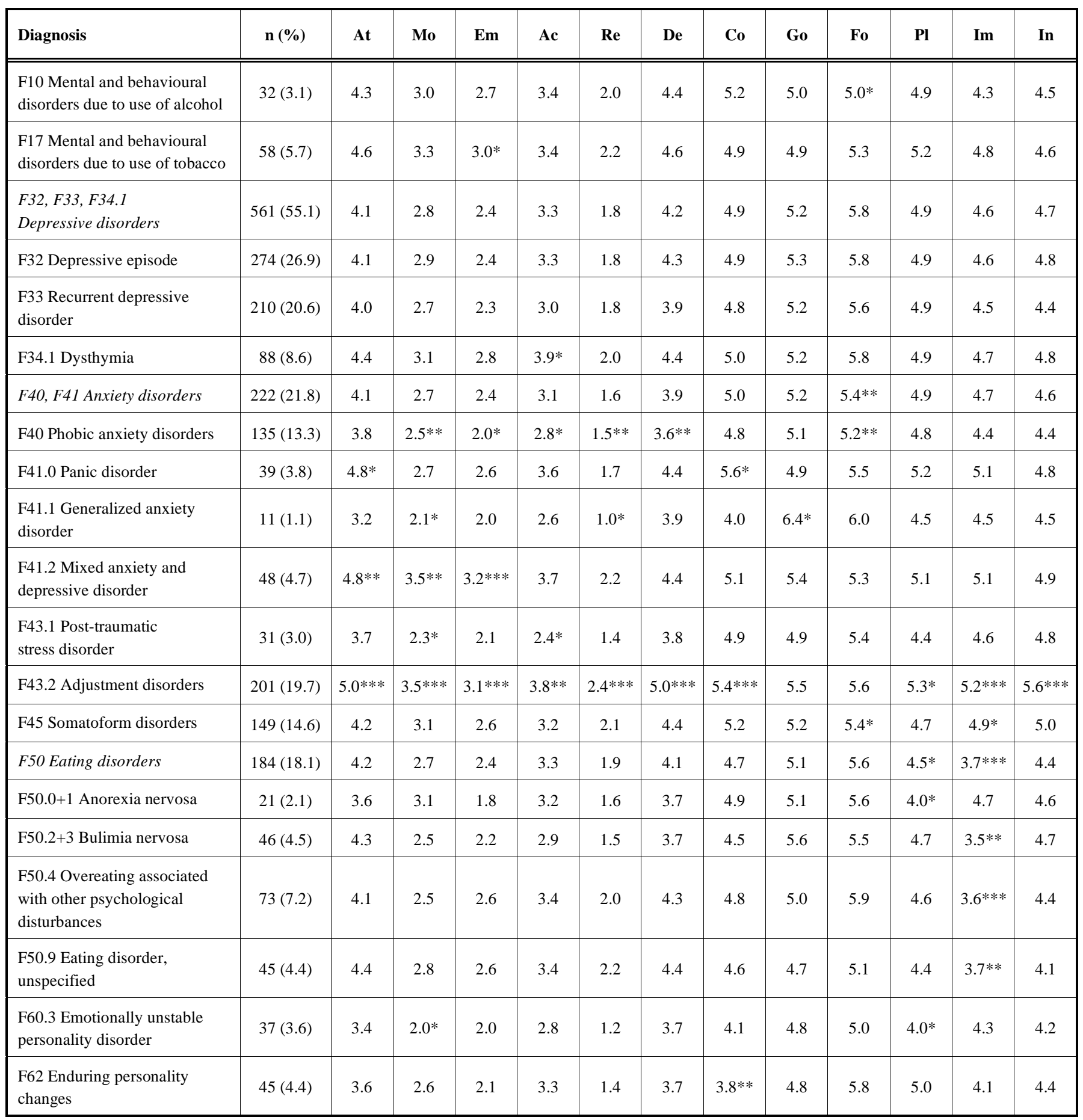

Notes: At: Attentional focusing. Mo: Self-motivation. Em: Emotion regulation. Ac: Self-activation. Re: Self-relaxation. De: Decision regulation. Co: Coping with failure. Go: Goal recollection. Fo: Forgetfulness prevention. Pl: Planning skill. Im: Impulse control. In: Initiating control.

$* p<.05 . * * p<.01$. *** $p<.001$. Significant differences of means between the subsamples with the respective diagnosis and the subsample with a depressive disorder (F32, F33, F34.1).

factor model with the 6 factors of the EFA (Table 5); (3) a 2factor model with the two macrocomponents self-regulation and self-control; and (4) a 1-factor model. All models produced a statistically significant $\chi^{2}$ value (see Table 6), indicating that the observed and specified models differed. However, this is not uncommon for large samples and does not necessarily reflect a poor fit to the data [50]. Based on the
RMSEA values reported in Table 6, the 12-factor model showed a good fit to the data, the 6-factor model an adequate fit, and the other models an unacceptable fit. Based on the SRMR values, the 12 -factor and the 6 -factor model showed a good fit to the data, and the other models an adequate fit. The AIC and BIC corroborated the finding that the 12-factor model provided the best fit, followed by the 6-factor model. 
Table 5. Varimax Rotated Factor Structure of the VCQ-36

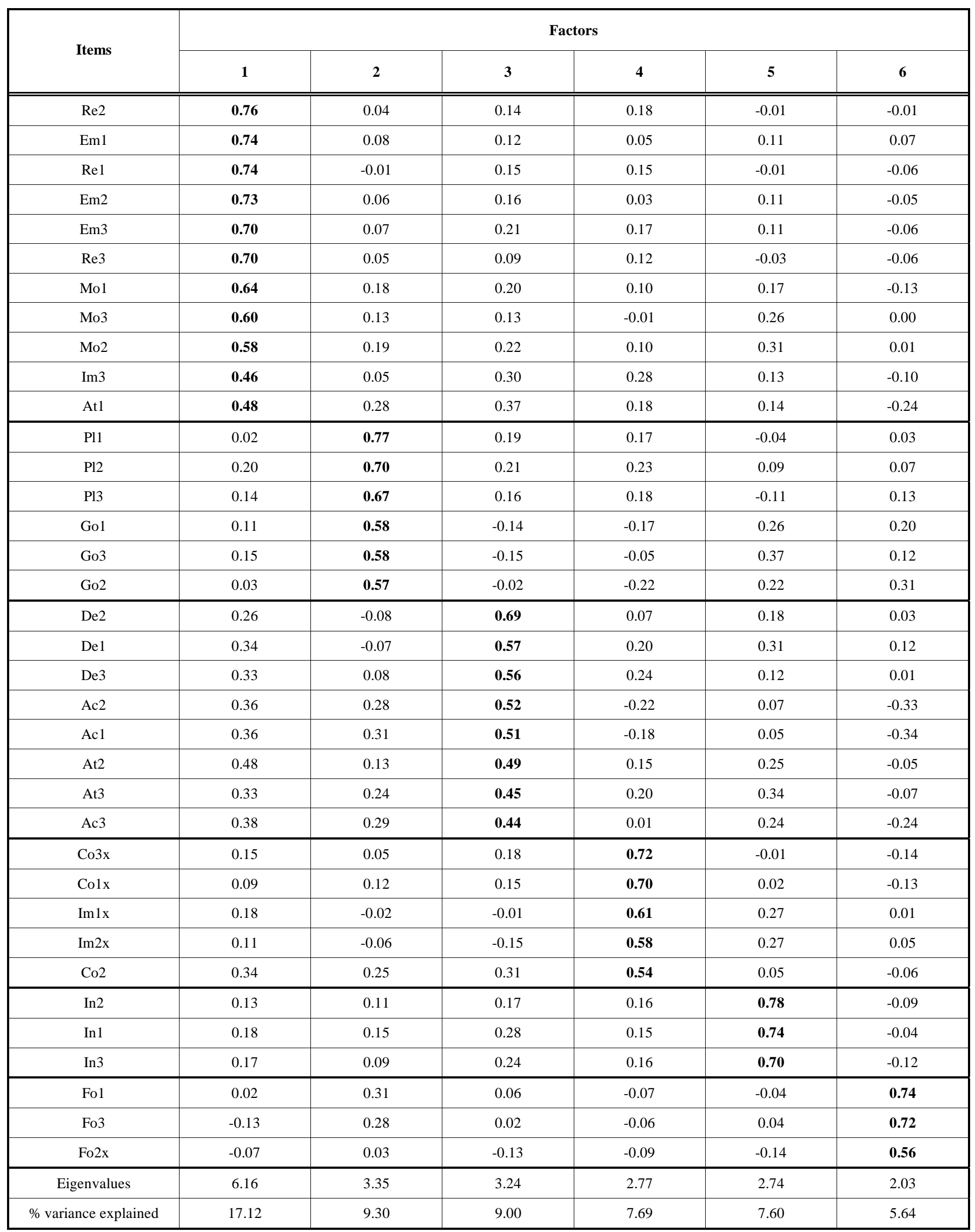

Notes: At: Attentional focusing. Mo: Self-motivation. Em: Emotion regulation. Ac: Self-activation. Re: Self-relaxation. De: Decision regulation. Co: Coping with failure. Go: Goal recollection. Fo: Forgetfulness prevention. Pl: Planning skill. Im: Impulse control. In: Initiating control. x: recoded. 
Table 6. Confirmatory Factor Analysis of the VCQ-36

\begin{tabular}{|c|c|c|c|c|c|c|c|}
\hline 1 factor & 7244.27 & 594 & $<.001$ & .105 & .069 & 7388.27 & 7742.91 \\
\hline 6 factors (resulting from EFA Table 5) & 2319.75 & 579 & $<.001$ & .077 & .059 & 2493.75 & 2861.97 \\
\hline
\end{tabular}

Note. SR: self-regulation (attentional focusing, self-motivation, emotion regulation, self-activation, self-relaxation, decision regulation, coping with failure); SC: self-control (goal recollection, forgetfulness prevention, planning skill, impulse control, initiating control).

\section{Relations to External Criteria}

\section{Psychopathology}

Table 7 presents the zero-order correlations computed to examine the relations of the volitional components to other constructs. Correlations with medium or high effect sizes are printed in bold. For the most part, self-regulation competences correlated negatively with depressive symptoms and total psychiatric symptomatology. Only one self-control competence, namely impulse control, correlated meaningfully with psychopathology. Physical complaints were negatively associated with self-relaxation competence only.

\section{Personality}

As expected, self-regulation competences correlated negatively with neuroticism, social inhibitedness, and excitability, and positively with extraversion. Impulse control was also negatively associated with neuroticism and excitability.
All self-regulation competences as well as initiating control correlated positively with achievement orientation.

\section{Cognitive Ability}

No meaningful correlation with cognitive ability was observed.

\section{DISCUSSION}

The results of the present study lend support to the reliability and validity of the VCQ-36. The internal consistencies of the VCQ-36 scales were satisfactory (Table 3), and the Cronbach's $\alpha$ coefficients were similar to those obtained by Kuhl and Fuhrmann [27]. The confirmatory factor analyses clearly showed that the VCQ-36 shared several volitional components with the original VCQ. Estimates of the models' fit to the data suggested that a 12 -factor model (i.e., the original 12 scales of the VCQ-36) was preferable to the 6factor model resulting from our exploratory factor analysis.

Table 7. Relationships of VCQ-36 Scales to Psychopathology, Personality, and Cognitive Ability $(\mathbf{N}=1018)$

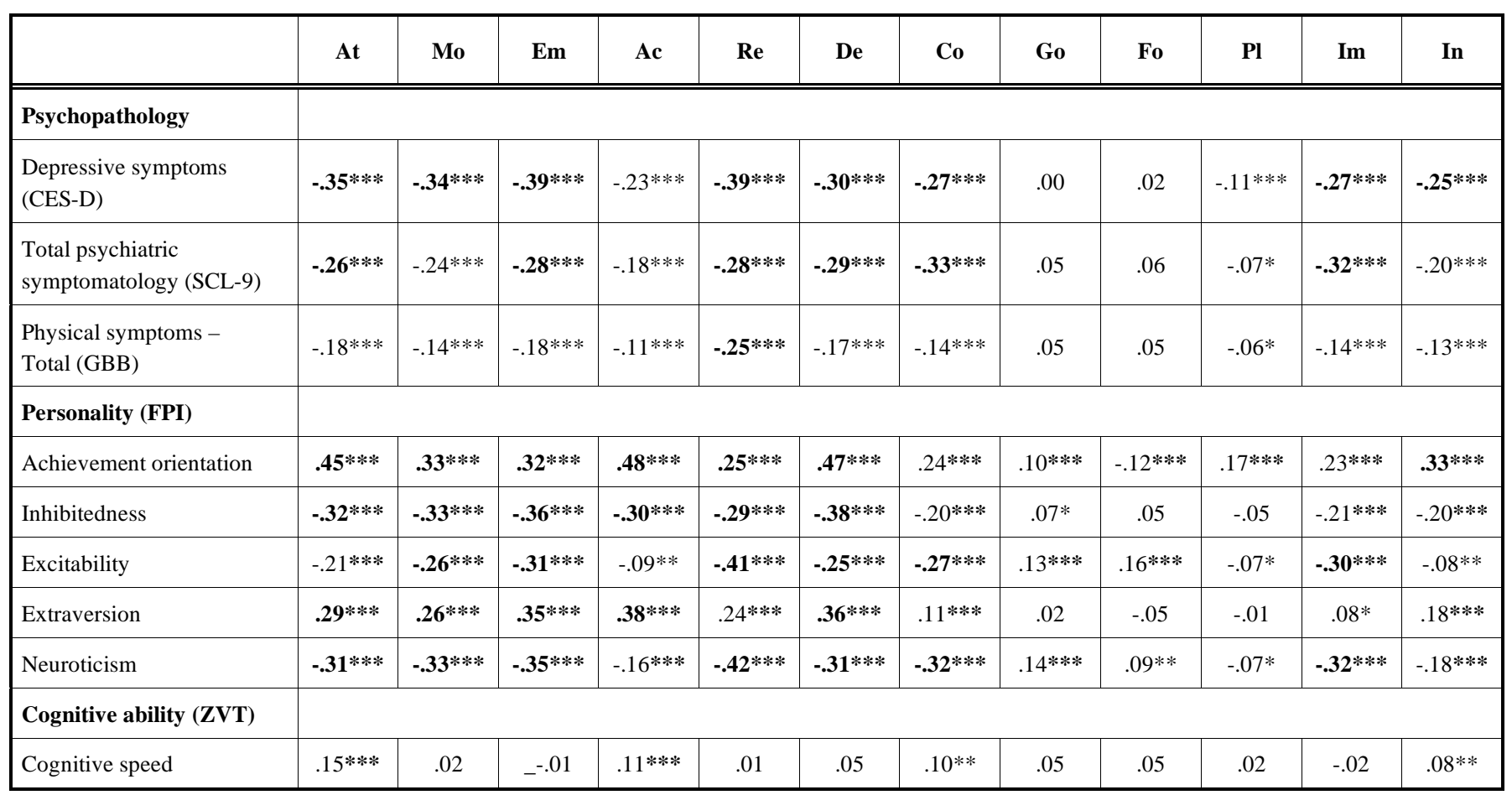

Note. Zero-order correlations. At $=$ attentional focusing, $\mathrm{Mo}=$ self-motivation, $\mathrm{Em}=$ emotion regulation, $\mathrm{Ac}=$ self-activation, $\mathrm{Re}=$ self-relaxation, $\mathrm{De}=$ decision regulation, $\mathrm{Co}=$ coping with failure, $\mathrm{Go}=$ goal recollection, $\mathrm{Fo}=$ forgetfulness prevention, $\mathrm{Pl}=$ planning skill, $\mathrm{Im}=$ impulse control, $\mathrm{In}=$ initiating control.

$* p<.05 . * * p<.01 . * * * p<.001$. Medium or high effect sizes $(r>=+-.25)$ are shown in bold. 
A two-factor solution with self-regulation and self-control competences as macrocomponents, as suggested by the theory of self-regulation [7, 8], was too approximate a classification of volition and did not satisfactorily represent the intercorrelations of the VCQ-36. Thus, we propose using the VCQ-36 primarily to measure the 12 volitional microcomponents and not the two macrocomponents. Clearly, researchers and practitioners interested in certain volitional competences can use the corresponding scales separately from the complete instrument [32, 33, 51, 52].

The EFA grouped most of the self-regulation competences into two factors, one combining skills involved in starting an action (decision, activation, and attention regulation), and one combining skills involved in influencing motivation and emotion (motivation, emotion, and relaxation regulation). These two sets of skills may be important in different action phases [12-14, 53]. The preparation phase requires self-control skills such as planning as well as selfregulation skills involved in decision making and initiating action. In the implementation phase, skills are needed that help to maintain motivation and to regulate emotion in order to reach a given goal.

The nomological net established by investigating the relation of the volitional competences with other constructs proved to be fairly meaningful. Most of the self-regulation competences correlated negatively with most of the psychopathological measures, whereas most of the self-control competences did not. This confirms the protective effect of self-regulation on mental and physical health that can be deduced from the theory of self-regulation [7,8] and previous research using the VCQ [28] and other measures [6, 11, $24,25]$. The most important volitional competences for mental health seem to be attentional focusing, self-motivation, emotion regulation, self-relaxation, decision regulation, coping with failure, and impulse control. The single most significant competence for physical health seems to be the ability for self-relaxation.

Impulse control showed the same correlational pattern as the self-regulation competences, although a different pattern had been expected. Forstmeier and Rüddel [38] found that patients using mainly self-regulation strategies have fewer depressive symptoms and less negative affect than patients using mainly self-control strategies. A core facet of selfcontrol is the suppression of unwanted impulses [9, 14]. Individuals who often suppress internal signals (emotions, needs, physical signs) experience more stress, negative emotions, and physiological activation, which may lead to a series of emotional and physical problems $[54,55]$. Two of the three impulse control items relate to resistance to temptation. This formulation is probably too global and does not differentiate very well between self-regulation and self-control. We propose developing some new items that are better capable of capturing the suppression aspect of self-control. It would also be desirable to include the suppression of emotions (e.g., by not expressing them), motivation (e.g., by ignoring competing goals), and attention (e.g., by stopping distracting stimuli). A good example for the measurement of the self-control and self-regulation aspects of emotion regulation is the Emotion Regulation Questionnaire (ERQ) [17]. The self-regulation items of the ERQ focus on cognitive reappraisal (e.g., "When I want to feel less negative emotion, I change the way I'm thinking about the situation"); the selfcontrol items are targeted at suppression of emotions (e.g., "When I am feeling negative emotions, I make sure not to express them").

The pattern of associations with personality variables confirms previous results obtained with the original VCQ [27]: self-regulation competences play a major role in emotional and social balance, showing negative associations with neuroticism, social inhibitedness, and excitability, and positive associations with extraversion. Although volitional competences were not found to influence performance in a cognitive speed task, as has been shown previously [27], they correlated highly with achievement orientation. This association might be the reason that people high in selfregulation receive higher school grades [11] and have higher occupational performance [33].

The fact that most volitional competences are similarly impaired in most psychiatric disorders implies that volitional disturbance is largely a common characteristic of mental impairment. This supports the findings obtained with the original VCQ [27]. However, there are also disorder-specific volitional impairments. Patients with phobic disorders appear to be highly impaired in self-motivation, emotion regulation, self-activation, self-relaxation, and decision regulation. This might reflect the avoidance symptoms of phobic disorders that hinder patients to effectively implement their chosen goals [29]. Patients with bulimia nervosa or overeating (but not anorexia nervosa) have particularly severe problems with impulse control, which is reflected in their recurrent failure to control impulses to eat. Finally, patients with adjustment disorders appear to be less volitionally impaired than most other subsamples. This is not surprising when considering the criteria of adjustment disorders, which exclude more severe disorders like depressive episode or anxiety disorders.

A number of limitations of the present study should be acknowledged. First, the sample consisted of patients with psychiatric or psychosomatic disorders. Mean values of the scales will probably be lower in a non-clinical sample. It would be desirable to replicate the present findings in a nonclinical sample to establish norms for the VCQ-36 scales. Second, the sample has a high proportion $(55 \%)$ of depressed patients and is therefore not representative of patients with mental disorders in general. However, as our results and those of others [27, 36] show, volitional competences are similarly impaired in most psychiatric disorders (see above). Third, this study applied a cross-sectional design. A longitudinal design is needed to test the assumption that volitional deficits are the causes of health and social problems. Forth, no alternative measure of volition was utilized to establish the convergent validity of the VCQ. In another study, however, Forstmeier and Maercker [51] reported high correlations between volitional measures. Finally, this study lacks behavioural measures of volition. However, Kuhl and Fuhrmann [27] and Fröhlich and Kuhl [36] reported significant associations of VCQ-based volitional measures and objective indices of volitional competence.

Taken together, the findings of the present study support the conclusion that volition is composed of several competences, and that the VCQ-36 is a reliable and valid instrument for assessing volitional competence. The VCQ-36 
draws a differentiated diagnostic picture of a person's volitional strengths and weaknesses, which might profitably be utilized in psychotherapy or educational/occupational training (e.g., [28, 33]). Other measures of volition offer only a global measure of volitional strength (e.g., ACS, LAQ, SCS) or single volitional competences (e.g., SRS, ERQ). Given that the original VCQ has proven sensitive to change [14], the VCQ-36 is presumably suitable for evaluating changes in volition after psychotherapy or training.

\section{REFERENCES}

[1] Metcalfe J, Mischel W. A hot/cool-system analysis of delay of gratification: Dynamics of willpower. Psychol Rev 1999; 106: 319.

[2] Lake B. Concept of ego strength in psychotherapy. Br J Psychiatry 1985; 147: 471-8.

[3] Kuhl J. Volitional mediators of cognition-behavior consistency: Self-regulatory processes and action versus state orientation. In: Kuhl J, Beckmann J, Eds. Action control: From cognition to behavior. Berlin: Springer 1985; pp. 101-28.

[4] Kuhl J. Action control: The maintenance of motivational states. In Halisch F, Kuhl J, Eds. Motivation, intention, and volition. Berlin: Springer 1987; pp. 279-91.

[5] Carver CS, Scheier MF. Control theory: A useful conceptual framework for personality, social, clinical and health psychology. Psychol Bull 1982; 92: 111-35.

[6] Kruglanski AW, Thompson EP, Higgins ET, et al. To "do the right thing" or to "just do it": Locomotion and assessment as distinct self-regulatory imperatives. J Pers Soc Psychol 2000; 79: 793-815.

[7] Kuhl J. A theory of self-regulation: Action versus state orientation, self-discrimination, and some applications. Appl Psychol Int Rev 1992; 1: 97-129.

[8] Kuhl J, Goschke T. A theory of action control: Mental subsystems, modes of control, and volitional conflict-resolution strategies. In: Kuhl J, Beckmann J, Eds. Volition and personality: Action versus state orientation. Göttingen: Hogrefe 1994; pp. 93-124.

[9] Kuhl J. A functional-design approach to motivation and selfregulation. In: Boekaerts M, Pintrich PR, Zeidner M, Eds. Handbook of self-regulation. San Diego: Academic Press 2000; pp. 111169.

[10] Schwarzer R. Optimism, goals, and threats: How to conceptualize self-regulatory processes in the adoption and maintenance of health behaviors. Psychol Health 1998; 13: 759-66.

[11] Tangney JP, Baumeister RF, Boone AL. High self-control predicts good adjustment, less pathology, better grades, and interpersonal success. J Pers 2004; 72: 271-322.

[12] Heckhausen H, Gollwitzer PM. Thought contents and cognitive functioning in motivational vs. volitional states of mind. Motiv Emot 1987; 11: 101-20.

[13] Heckhausen J, Heckhausen H. Motivation and action. New York: Cambridge University Press 2008.

[14] Kuhl J. The volitional basis of Personality Systems Interaction Theory: Applications in learning and treatment contexts. Int J Educ Res 2000; 33: 665-705.

[15] Green-Demers J, Pelletier LG, Stewart DG, Gushue NR. Coping with the less interesting aspects of training: Toward a model of interest and motivation enhancement in individual sports. Basic Appl Soc Psychol 1998; 20: 251-61.

[16] Mischel W. From good intentions to willpower. In: Gollwitzer PM, Bargh JA, Eds. The psychology of action: Linking cognition and motivation to behaviour. New York: Guilford Inc: 1997; pp. 197219.

[17] Gross JJ, John OP. Individual differences in two emotion regulation processes: Implications for affect, relationships, and wellbeing. J Pers Soc Psychol 2003; 85: 348-62.

[18] Gross JJ, Ed. Handbook of emotion regulation. New York: Guilford Press 2007.

[19] Morris WN, Reilly NP. Toward the self-regulation of mood: Theory and research. Motiv Emot 1987; 11: 215-49.

[20] Thayer RE, Newman JR, McClain TM. Self-regulation of mood: Strategies for changing a bad mood, raising energy, and reducing tension. J Pers Soc Psychol 1994; 67: 910-25.
[21] Westen D. Toward an integrative model of affect regulation: Applications to social-psychological research. J Pers 1994; 62: 641-67.

[22] Atkinson JW, Birch DA. A dynamic theory of action. New York: John Wiley 1970.

[23] Norman DA, Shallice T. Attention to action: Willed and automatic control of behavior. In: Davidson JE, Schwartz GE, Shapiro D, Eds. Conciousness and self-regulation: Advances in research. New York: Plenum Press 1985.

[24] Beckmann J, Kellmann M. Self-regulation and recovery: Approaching an understanding of the process of recovery from stress. Psychol Rep 2004; 95: 1135-53.

[25] Luszczynska A, Diehl M, Gutiérrez Doña B, Kuusinen P, Schwarzer R. Measuring one component of dispositional selfregulation: Attention control in goal pursuit. Pers Individ Dif 2004; 37: 555-66.

[26] Hautzinger M. Action control in the context of psychopathological disorders. In: Kuhl J, Beckmann J, Eds. Volition and personality: Action versus state orientation. Göttingen: Hogrefe 1994; pp. 20915 .

[27] Kuhl J, Fuhrmann A. Decomposing self-regulation and selfcontrol: The Volitional Components Inventory. In: Heckhausen J, Dweck CS, Eds. Motivation and self-regulation across the life span. Cambridge: Cambridge University Press 1998; pp. 15-49.

[28] Forstmeier S, Rüddel H. Improving volitional competence is crucial for the efficacy of psychosomatic therapy: A controlled clinical trial. Psychother Psychosom 2007; 76: 89-96.

[29] Hartung J, Schulte D. Action and state orientation during therapy of phobic disorders. In: Kuhl J, Beckmann J, Eds. Volition and personality: Action versus state orientation. Göttingen: Hogrefe 1994.

[30] Rholes WS, Michas L, Shroff J. Action control as a vulnerability factor in dysphoria. Cognit Ther Res 1989; 13: 263-74.

[31] Finkel EJ, Campbell WK. Self-control and accommodation in close relationships: An interdependence analysis. J Pers Soc Psychol 2000; 81: 263-77.

[32] Orbell S. Personality systems interactions theory and the theory of planned behaviour: Evidence that self-regulatory volitional components enhance enactment of studying behaviour. Br J Soc Psychol 2003; 42: 95-112.

[33] Kehr HM, Bles P, von Rosenstiel L. Self-regulation, self-control, and management training transfer. Int J Educ Res 1999; 31: 487-98.

[34] Kuhl J. Action versus state orientation: Psychometric properties of the Action Control Scale (ACS-90). In: Kuhl J, Beckmann J, Eds. Volition and personality: Action versus state orientation. Göttingen: Hogrefe 1994.

[35] Kaschel R, Kuhl J. Motivational counseling in an extended functional context: Personality Systems Interaction Theory and assessment. In: Cox WM, Klinger E, Eds. Handbook of motivational counseling: Motivating people for change. Sussex: Wiley 2004.

[36] Fröhlich SM, Kuhl J. The Volitional Components Inventory [Das Selbststeuerungsinventar: Dekomponierung volitionaler Funktionen]. In: Stiensmeier-Pelster J, Rheinberg F, Eds. Diagnostik von Motivation und Selbstkonzept. Göttingen: Hogrefe 2003; pp. 22157.

[37] Fuhrmann A, Kuhl J. Maintaining a healthy diet: Effects of personality and self-reward versus self-punishment on commitment to and enactment of self-chosen and assigned goals. Psychol Health 1998; 13: 651-86.

[38] Forstmeier S, Rüddel H. On the superiority of self-regulation over self-control in psychotherapy and psychosomatic rehabilitation. Verhaltensther 2005; 15: 158-66.

[39] World Health Organization. The ICD-10 classification of mental and behavioural disorders: Clinical descriptions and diagnostic guidelines. Geneva: WHO 1992.

[40] Radloff LS. The CES-D scale: A self-report depression scale for research in the general population. Appl Psychol Meas 1977; 3: 385-401.

[41] Hautzinger M, Bailer M. Allgemeine Depressionsskala (ADS). Weinheim: Beltz 1993

[42] Klaghofer R, Brähler E. Construction and test-statistical examination of a short form of the SCL-90-R [Konstruktion und teststatistische Prüfung einer Kurzform der SCL-90-R]. Z Klin Psychol Psychiatr Psychother 2001; 49: 115-24.

[43] Derogatis LR. SCL-90-R, administration, scoring \& procedures manual-I for the revised version. Baltimore: Johns Hopkins University School of Medicine 1977. 
[44] Brähler E, Scheer JW. Giessen Subjective Complaints List [Giessener Beschwerdebogen]. Bern: Huber 1995.

[45] Fahrenberg J, Hampel R, Selg H. Freiburg Personality Inventory Manual [Freiburger Persönlichkeitsinventar - Handanweisung]. Göttingen: Hogrefe 2001.

[46] Oswald WD, Roth E. Digit Connection Test [Der ZahlenVerbindungs-Test (ZVT)]. Göttingen: Hogrefe 1987.

[47] Horn JL. A rationale and test for the number of factors in factor analysis. Psychometrika 1965; 30: 179-85.

[48] O'Connor BP. SPSS and SPS programs for determining the number of components using parallel analysis and Velicer's MAP test. Behav Res Methods Instrum Comput 2000; 32: 396-402.

[49] Hu L, Bentler PM. Cutoff criteria for fit indexes in covariance structure analysis: Conventional criteria versus new alternatives. Struct Equation Model 1999; 6: 1-55.

[50] Bollen K. Structural equations with latent variables. New York: John Wiley 1989.
[51] Forstmeier S, Maercker A. Motivational reserve in dementia: Development of an assessment concept [Motivationale Reservekapazität bei dementiellen Erkrankungen: Entwicklung eines Messkonzeptes]. Nervenarzt 2005; 76(Suppl. 1): S100-1.

[52] Forstmeier S, Maercker A. Motivational reserve: lifetime motivational abilities influence cognitive and emotional health in old age. Psychol Aging (in press).

[53] Prochaska JO, DiClemente CC. Transtheoretical therapy: Toward a more integrative model of change. Psychother Theory Res Pract 1982; 19: 276-88.

[54] Pennebaker JW. Traumatic experience and psychosomatic disease: Exploring the roles of behavioural inhibition, obsession, and confiding. Can Psychol 1985; 26: 82-95.

[55] Polivy J. Inhibition of internally cued behavior. In: Higgins ET, Sorrentino RM, Eds. Handbook of motivation and cognition: Foundations of social behavior, Vol. 2. New York 1990; pp. 131-47.

Received: March 24, 2008

Revised: June 21, 2008

Accepted: July 25, 2008

(c) Forstmeier and Rüddel; Licensee Bentham Open.

This is an open access article licensed under the terms of the Creative Commons Attribution Non-Commercial License (http://creativecommons.org/licenses/ by-nc/3.0/) which permits unrestricted, non-commercial use, distribution and reproduction in any medium, provided the work is properly cited. 